\title{
Comportamento dos Custos das Empresas Brasileiras do Segmento de Energia Elétrica Listadas na BM\&FBOVESPA entre o Período de 2006 e 2015
}

\begin{abstract}
Monalisa de Castro Souza
Graduação em Ciências Contábeis pela Universidade Federal de Uberlândia -

UFU

Contadora Autônoma

E-mail:monacastrosouza@hotmail.com

Edvalda Araújo Leal

Doutorado em Administração pela Fundação Getúlio Vargas - FGV

Professora da Universidade Federal de Uberlândia - UFU

Av. João Naves de Ávila, 2121. Santa Mônica, Uberlândia/MG. Brasil. CEP: 38.400-902

E-mail: edvalda@facic.ufu.br
\end{abstract}

\section{RESUMO}

O presente trabalho buscou analisar o comportamento dos custos das empresas brasileiras do segmento de energia elétrica listadas na BM\&FBOVESPA, entre os anos de 2006 e 2015. A pesquisa classifica-se como descritiva, com abordagem quantitativa. Para os procedimentos de coleta de dados, utilizou-se a pesquisa documental, visto que foram utilizados os demonstrativos financeiros das empresas do segmento de energia elétrica. A coleta dos dados foi realizada na base de dados do Economática. A amostra foi composta por 17 empresas, das quais se coletaram, a partir dos demonstrativos contábeis, as informações referentes a: Receita Líquida de Vendas RLV, Custo do Produto Vendido - CPV, Despesas Administrativas - DA e Despesas com Vendas - DV. Os resultados demonstraram que, no segmento de energia elétrica, o CPV consome, em média, $56 \%$ da RLV, as despesas com vendas cerca de $2 \%$ e as despesas administrativas consomem, aproximadamente, $21 \%$ da RLV. Esse resultado não se mostrou correlato com os resultados encontrados em outros setores, em que a parcela da RLV consumida pelo CPV foi, em média, de $75 \%$. As variações percentuais da RLV e do CPV foram convergentes e positivas na maioria das empresas analisadas; já os aumentos na RLV refletiram-se em aumentos também no CPV. Esse resultado é compatível com a correlação forte e positiva encontrada entre as variáveis RLV e CPV, indicando que, no segmento analisado, aumentos da RLV indicam aumentos do CPV.

Palavras-chave: Comportamento dos Custos. Energia Elétrica. BM\&FBOVESPA. Segment at the BM\&FBOVESPA between the Period 2006 and 2015 


\section{ABSTRACT}

This study aimed to analyze the cost behavior of Brazilian companies in the electricity segment listed on BM \& FBOVESPA, from 2006 to 2015. The research is classified as descriptive, with quantitative approach. For the data collection procedures, we used the document research, since the financial statements of companies in the electricity sector were used. Data collection was performed in Economática database. The sample consisted of 17 companies, which were collected from the financial statements, information regarding: Net Sales - RLV, Cost of Goods Sold - CPV, Administrative Expenses - DA and Selling Expenses - DV. The results showed that, in the electricity segment, CPV consumes on average $56 \%$ of the RLV, selling expenses about $2 \%$ and administrative costs consume about $21 \%$ of the RLV. This result was not correlative with the results found in other sectors, which the share of RLV consumed by the CPV was on average $75 \%$. The percentage changes of RLV and CPV were converging and positive in most of the analyzed companies; the increase in RLV reflected in an increase in CPV as well. This result is compatible with the strong positive correlation between the RLV and CPV variables, indicating that, in the analyzed segment, increases of RLV indicate increases of CPV.

Keywords: Cost Behavior. Electricity. BM\&FBOVESPA.

\section{INTRODUÇÃO}

De acordo com o relatório de Matriz Energética Brasileira 2030, estima-se o consumo total de energia elétrica, em 2030, em 1.030,1 TWh, ficando as perdas na ordem de 13,8\%. A oferta interna, em 2030, é calculada em 1.194,9 TWh, e a produção, em 1.151,0 TWh. O mesmo relatório apresenta o consumo dos setores da economia para 2030 , continuando o setor industrial a ser o principal consumidor, com $42 \%$, com destaque para o setor terciário, que se apresenta responsável por $25 \%$ do consumo. Diante desse contexto, torna-se necessário elaborar uma estratégia para atendimento das demandas, como, por exemplo, iniciativas na área de eficiência energética (Brasil, 2007).

Nesse cenário, as empresas do setor de energia carecem cada vez mais de inovação para atender as necessidades dos clientes. Assim, segundo Rabelo (2012), é indispensável que o gestor tenha conhecimento dos custos e do modo como eles se 
comportam e, além disso, que detenha as informações do mercado para que possa agir eficientemente, visando ao sucesso e aumento dos resultados empresariais.

Nesse sentido, ao conhecerem o modo como os custos se comportam, os gestores podem adequar suas atividades a fim de maximizarem os lucros, visto que, dessa maneira, teriam condições de antecipar os melhores caminhos nas mais diferentes situações empresariais (Medeiros, Costa, \& Silva, 2005).

Diante do exposto, a pergunta de pesquisa deste estudo é: Como se comportaram os custos das empresas brasileiras do segmento de Energia Elétrica listadas na BM\&FBOVESPA, no período de 2006 a 2015? Sendo assim, o objetivo geral da pesquisa é analisar o comportamento dos custos em relação às receitas líquidas de vendas das empresas brasileiras do segmento de energia elétrica listadas na BM\&FBOVESPA entre 2006 e 2015.

Um estudo sobre comportamento dos custos se justifica, pois as informações acerca dos custos empresariais são necessárias para o atendimento dos interesses dos usuários da contabilidade, seja para a tomada de decisão dos gerentes e/ou para o conhecimento dos usuários externos acerca do consumo da receita pelos custos, bem como acerca das oscilações dos custos em relação às variações da receita ao longo dos anos.

Poucos foram os estudos que trataram de segmentos específicos a fim de analisar o comportamento dos custos. Os segmentos de Fios e Tecidos e de Telecomunicações, além dos setores Aéreo, Petroquímico e de Carnes e Derivados foram temas de estudos que analisaram o comportamento dos custos no Brasil. Ademais, nenhum trabalho buscou analisar o comportamento dos custos no segmento de energia elétrica. Assim, o presente trabalho busca analisar como se comportam os custos em relação às receitas das empresas brasileiras listadas no segmento de energia elétrica da BM\&FBOVESPA.

Espera-se, por meio do presente estudo, contribuir com a análise do comportamento dos custos nas empresas do segmento de energia elétrica, visto que essas informações poderão auxiliar os vários usuários da contabilidade no processo de 
Comportamento dos Custos das Empresas Brasileiras do Segmento de Energia Elétrica Listadas na BM\&FBOVESPA entre o Período de 2006 e 2015 Monalisa de Castro Souza, Edvalda Araújo Leal

decisão, no desenvolvimento de pesquisas e na geração de conhecimento científico na área.

\section{REFERENCIAL TEÓRICO}

O referencial teórico subdivide-se em dois tópicos: primeiramente, será apresentada a caracterização do segmento de energia elétrica e, posteriormente, serão apresentados conceitos e resultados de pesquisas acerca do tema comportamento dos custos, bem como a exposição sobre o comportamento assimétrico dos custos (a teoria dos Sticky Costs) evidenciada na literatura pesquisada.

\subsection{Contextualização do Segmento de Energia Elétrica}

A energia elétrica é elemento fundamental para a execução das atividades humanas, sendo imprescindível para o desenvolvimento socioeconômico do país. Esse setor de Energia Elétrica é formado por quatro segmentos: geração, transmissão, distribuição e comercialização (Andrade, 2015).

A regulação e a fiscalização do setor de energia elétrica são de responsabilidade da Agência Nacional de Energia Elétrica [ANEEL], autarquia vinculada ao Ministério de Minas e Energia e instituída pela Lei $n^{\circ} 9.427 / 1996$ e pelo Decreto $n^{\circ} 2.335 / 1997$. Suas principais atribuições são: regular a geração, transmissão, distribuição e comercialização de energia elétrica; fiscalizar e promover as concessões, permissões e serviços de energia elétrica; estabelecer tarifas; e mediar conflitos diversos (Agência Nacional de Energia Elétrica [ANEEL], (2015a).

De acordo com o Boletim de Informações Gerenciais da ANEEL (2015b), o Brasil possui 4.405 usinas em operação, que correspondem a uma potência instalada de 140.271.642 kW. Do total de usinas, as termelétricas representam $64,8 \%$, porém essas têm somente $39.392 .797 \mathrm{~kW}$ de capacidade instalada. As usinas hidrelétricas representam $4,5 \%$ do total, com potência instalada de $86.002 .478 \mathrm{~kW}$. 
As fontes em operação utilizadas no Brasil são, na maioria, hídricas (65,44\%). Já as outras fontes podem ser assim descritas: a fóssil representa 18,90\% (carvão mineral, gás natural, petróleo, entre outros); a biomassa, 9,57\% (bagaço de cana de açúcar, óleos vegetais, entre outros); a eólica, 4,71\%; a nuclear, 1,37\%; e a solar, 0,01\% (ANEEL, 2015b).

Segundo dados da ANEEL (2015b), a matriz de energia elétrica no Brasil é composta, em sua maioria, pela energia hidrelétrica $(61,77 \%)$, representando a fóssil 17,74\%, a biomassa, 8,96\%, a importação, 5,55\% (Paraguai, Argentina, Venezuela e Uruguai), a eólica, $4,60 \%$, a nuclear, $1,37 \%$ e a solar, $0,01 \%$.

O Boletim de Informações Gerenciais divulgado pela ANEEL elenca os dez maiores agentes de distribuição, por receita de fornecimento, representando $58,2 \%$ do total nacional. Esse percentual totaliza uma receita de fornecimento de Energia Elétrica de $\mathrm{R} \$ 77.720 .473 .655,83$ no ano de 2015.

De acordo com o balanço energético nacional divulgado pelo Ministério de Minas e Energia (ANEEL, 2015c), a oferta interna de energia elétrica, em 2014, foi de 624,3 TWh (2,1\% superior a 2013), sendo 33,8 TWh de importações líquidas e 590,5 TWh de geração em centrais de serviço público e autoprodutores (geração 3,4\% superior a 2013). O consumo final foi de $531,1 \mathrm{TWh}$, nesse mesmo ano (acréscimo de $2,9 \% \mathrm{em}$ relação a 2013).

O relatório de Matriz Energética Brasileira 2030 traz projeções de oferta e demanda de energia no Brasil até o ano de 2030, onde nota-se um crescimento no consumo residencial de $19 \%$, em 2005 , para $23 \%$, em 2030 , o que pode ser explicado pelo aumento de renda da população, gerando acesso à energia e melhoria na distribuição (Brasil, 2007).

O Boletim de Informações Gerenciais divulgado pela ANEEL (2015b) apresenta, conforme Tabela 1, a evolução da receita, do consumo e das unidades consumidoras, no período de 2005 a 2015. Com relação ao consumo, houve queda de $-0,8 \%$ no ano de 2015, tendo o maior percentual de aumento ocorrido em 2008 (5,8\%). A receita de fornecimento obteve retração de $-10,6 \%$, em 2013 , e maior percentual de crescimento, 
Comportamento dos Custos das Empresas Brasileiras do Segmento de Energia Elétrica Listadas na BM\&FBOVESPA entre o Período de 2006 e 2015 Monalisa de Castro Souza, Edvalda Araújo Leal

em 2015 (40\%). Nas unidades consumidoras, ocorreram quedas no crescimento desde o ano de 2009 (ANEEL, 2015b).

Tabela 1

Receita e Consumo - mercado cativo

\begin{tabular}{|c|c|c|c|c|c|c|}
\hline Ano & $\begin{array}{c}\text { Consumo de } \\
\text { energia elétrica } \\
\text { (Mwh) }\end{array}$ & $\begin{array}{c}\% \\
\text { Cresc. }\end{array}$ & $\begin{array}{c}\text { Receita de } \\
\text { fornecimento de } \\
\text { energia elétrica }\end{array}$ & $\begin{array}{c}\% \\
\text { Cresc. }\end{array}$ & $\begin{array}{c}\text { Número de } \\
\text { unidades } \\
\text { consumidoras }\end{array}$ & $\begin{array}{c}\% \\
\text { Cresc. }\end{array}$ \\
\hline 2005 & $253.260 .853,86$ & & $\mathrm{R} \$ 61.426 .214 .861,28$ & & 56.836 .625 & \\
\hline 2006 & $252.107 .694,46$ & $-0,5 \%$ & $\mathrm{R} \$ 64.706 .373 .433,80$ & $5,3 \%$ & 58.261 .836 & $2,50 \%$ \\
\hline 2007 & $263.215 .700,26$ & $4,4 \%$ & $\mathrm{R} \$ 68.122 .958 .570,12$ & $5,3 \%$ & 60.534 .375 & $3,90 \%$ \\
\hline 2008 & $278.592 .020,41$ & $5,8 \%$ & $\mathrm{R} \$ 68.838 .701 .970,26$ & $1,1 \%$ & 63.088 .315 & $4,20 \%$ \\
\hline 2009 & $286.871 .823,71$ & $3,0 \%$ & $\mathrm{R} \$ 74.456 .147 .481,54$ & $8,2 \%$ & 65.450 .236 & $3,70 \%$ \\
\hline 2010 & $302.398 .063,14$ & $5,4 \%$ & $\mathrm{R} \$ 80.005 .116 .616,98$ & $7,5 \%$ & 67.627 .240 & $3,30 \%$ \\
\hline 2011 & $310.398 .063,14$ & $2,6 \%$ & $\mathrm{R} \$ 86.435 .093 .265,57$ & $8,0 \%$ & 70.130 .344 & $3,70 \%$ \\
\hline 2012 & $319.871 .062,06$ & $3,1 \%$ & $\mathrm{R} \$ 93.675 .397 .987,68$ & $8,4 \%$ & 72.245 .611 & $3,00 \%$ \\
\hline 2013 & $329.429 .678,77$ & $3,0 \%$ & $\mathrm{R} \$ 83.711 .095 .870,92$ & $-10,6 \%$ & 74.669 .108 & $3,40 \%$ \\
\hline 2014 & $345.223 .238,50$ & $4,8 \%$ & $\mathrm{R} \$ 95.368 .593 .165,50$ & $13,9 \%$ & 76.883 .226 & $3,00 \%$ \\
\hline 2015 & $342.346 .955,34$ & $-0,8 \%$ & $\mathrm{R} \$ 133.555 .897 .959,26$ & $40,0 \%$ & 78.941 .194 & $2,70 \%$ \\
\hline
\end{tabular}

Nota. Fonte: Elaborado pela autora, com base em ANEEL (2015b).

A seguir, são apresentados estudos relacionados ao comportamento dos custos, seja analisando conjuntamente empresas de diversos segmentos ou apenas um segmento específico.

\subsection{Comportamento dos Custos e a relação com a teoria de Sticky Costs}

O gerenciamento dos custos implica melhores e contínuos controles dos processos, bem como aumento dos resultados e do nível competitivo das organizações (Duarte, Fehr, Tavares, \& Reis, 2013). Para os gestores, entender o comportamento dos custos implica antecipar obstáculos, buscando, com isso, melhor planejamento dos processos da organização (Medeiros et al., 2005).

De acordo com Padoveze (2009), "entende-se por comportamento dos custos a evolução de como tais gastos acontecem em alguma relação com dados físicos de 
produção, venda ou outra atividade". Para complementar, Gomes, Lima e Steppan (2007) afirmam que "o comportamento dos custos descreve a maneira como os mesmos se alteram a partir de mudanças nos níveis de atividade ou no volume de produção, de modo a minimizar as dúvidas de como os custos são gerados quando as atividades são desempenhadas".

Assim, comportamento dos custos se define como a forma de variação dos custos devido a modificações no volume de atividades e vendas, bem como na estrutura empresarial, sofrendo influências do ambiente, da sociedade e da economia (Richartz, Borgert, \& Lunkes, 2014).

Anderson, Banker e Janakiraman (2003) desenvolveram a Teoria dos sticky costs (assimetria dos custos) para explicar esta variação. Esta teoria defende que "os custos são assimétricos se a magnitude do aumento dos custos associados com um aumento em volume for maior do que a magnitude da diminuição nos custos associados com a redução equivalente em volume" (Anderson, Banker \& Janakiraman, 2003).

Segundo Richartz, Borgert e Ensslin (2014), as primeiras publicações a respeito desse tema remontam à década de 1960, com o artigo de Benston, "Multiple Regression Analysis of Cost Behavior", publicado no periódico "The Accounting Review", em 1966. Esses estudos evidenciavam a simetria da relação entre custos e volume. Dessa forma, de acordo com as alterações no nível de atividade, os custos são classificados em fixos e variáveis, ou seja, os custos se alteram com base no volume de atividades, sem a preocupação com o sentido da mudança (Noreen, 1991).

De acordo com essa abordagem, denominada tradicional, os custos variáveis são aqueles que se alteram conforme o nível de produção. Já os custos fixos não se alteram com o volume produzido, pois eles ocorrem independentemente de haver ou não produção (Martins, 2003).

Essas definições são feitas com base no total, já que o comportamento desses custos tomados unitariamente tornam-se os custos fixos variáveis por unidade e os custos variáveis fixos por unidade (Oliveira, Lustosa, \& Sales, 2007). 
Pode-se destacar que os custos fixos e variáveis podem ser diretos ou indiretos. Os custos diretos são aqueles de fácil identificação quanto aos produtos, ou seja, podem ser destinados de forma direta a cada produto. Diferentemente, os custos indiretos não podem ser identificados por produto e, sim, são comuns a toda a produção (Padoveze, 2009).

Estudos recentes indicaram que os custos não se alteram somente com base no nível de produção, mas também consideram o impacto do sentido da variação, aumento ou diminuição da receita (Richartz et al., 2014). O estudo que alterou o entendimento da abordagem tradicional de que os custos se alteram somente conforme a variação da receita, sem a preocupação com o sentido dessa variação (aumento ou diminuição), foi o de Anderson, Banker e Janakiraman (2003).

No referido estudo, os autores verificaram, com base em uma amostra de 7.629 empresas, ao longo de 20 anos, que o comportamento dos custos não depende somente das variações da receita, mas também do aumento ou redução da mudança.

Assim, os custos aumentam mais quando as receitas aumentam do que diminuem quando as receitas diminuem na mesma proporção. Essa é a teoria chamada Sticky Costs, cuja premissa é de que os custos são assimétricos à receita, ou seja, não variam na mesma intensidade das receitas (Anderson et al., 2003).

Alguns estudos foram realizados para testar a teoria dos Sticky Costs, cujas discussões foram iniciadas por Anderson et al. (2003).

Medeiros et al. (2005) utilizaram uma amostra de 198 empresas, no período de 17 anos, e confirmaram que a teoria dos Sticky Costs é parcialmente aplicável ao Brasil. Os autores constataram ainda que a assimetria não diminui quando considerados períodos maiores que um exercício, mas confirmou-se uma reversão parcial quando considerados períodos defasados.

Com o propósito de analisar o comportamento de custos, Richartz et al. (2014) analisaram 136 empresas, ao longo de 10 anos, para identificar se os gastos com mão de obra e custos fixos tinham relação com a assimetria dos custos. Os autores 
confirmaram a assimetria dos custos para as empresas brasileiras, porém não encontraram influências dos gastos com os Sticky Costs.

Richartz e Borgert (2014) utilizaram uma amostra das empresas brasileiras listadas na BM\&FBOVESPA, entre 1994 e 2011, e comprovaram que o custo dos produtos vendidos (CPV) apresentou queda ao longo dos períodos, havendo ainda melhoria da eficiência operacional. Com relação aos Sticky Costs, para variações na receita de até $10 \%$, a teoria se confirma, ou seja, para cada $1 \%$ de aumento na receita líquida de vendas (RLV), o CPV aumenta 0,96\%, e quando a RLV diminui 1\%, o CPV diminui apenas 0,92\%. Entretanto, para variações na receita de mais de $10 \%$, ocorre o inverso, ou seja, se a RLV diminui 1\%, o CPV reduz 0,89\%, e se a RLV aumenta 1\%, o CPV aumenta 0,83\%.

Envolvendo empresas internacionais, Costa, Marques, Santos e Lima (2013) analisaram uma amostra de 669 empresas abertas de nove países da América Latina, durante o período de 1995 a 2012. Os autores verificaram a existência de assimetria das despesas de vendas gerais e administrativas (VGA) em relação às variações na RLV. As VGAs aumentam 0,56\% para 1\% de aumento na RLV e diminuem 0,45\% para uma mesma redução na RLV.

Já Richartz, Borget, Vicente e Ferrara (2012) utilizaram uma amostra de 14 empresas do segmento de fios e tecidos, listadas na BM\&FBOVESPA, entre 1998 e 2010, e constataram que, em média, 78\% da RLV é consumida pelo CPV. As despesas com vendas representaram $10 \%$ da $R L V$; as despesas financeiras apresentaram variabilidade somente em certo período; e as despesas administrativas não apresentaram tendência de crescimento. Outra comprovação dos autores é que as empresas com menor receita apresentaram melhor relação CPV/RLV. Com relação à análise dos Sticky Costs, para variações na RLV de até 30\%, a assimetria dos custos é constatada, porém para variações acima de 30\%, consequentemente, a teoria é refutada.

Fazoli, Reis e Borgert (2015) constataram, com base em uma amostra de 7.760 empresas industriais com sede em Santa Catarina, que a teoria dos Sticky Costs é 
aplicável ao setor de forma geral, tendo os custos aumentados em $0,7781 \%$, quando ocorreu aumento de $1 \%$ nas receitas líquidas de vendas, enquanto que, para uma mesma diminuição nas receitas, os custos diminuíram em $0,7632 \%$. Os autores ainda separaram as indústrias em 24 setores e verificaram que, em sua maioria (12 setores), o comportamento apresentado corrobora a teoria proposta por Anderson et al. (2003). Dos demais, 8 setores apresentaram comportamento Anti-Stick e 4 setores tiveram variações simétricas.

Lopes e Beuren (2015) identificaram, no setor aéreo brasileiro, assimetria dos custos. Para um acréscimo de $1 \%$ nas receitas, os custos aumentam $0,86 \%$ e, para um decréscimo de igual percentual nas receitas, os custos se reduzem em 0,59\%. Relacionando o comportamento dos custos com medidas não financeiras de desempenho, número de empregados e aeronaves, verificou-se influência no comportamento dos custos.

Outros estudos trataram do comportamento dos custos sem abordar a teoria dos Sticky Costs, mas relacionando-os com diferentes variáveis, como Oliveira et al. (2007), que comprovaram a melhoria da eficiência produtiva após a privatização, em comparação ao período anterior de estatização da Companhia Vale do Rio Doce. Para essa análise, foram separados os custos fixos e variáveis por meio de regressão linear simples, cujas informações de CPV e receita operacional líquida $(R O L)$ foram retiradas dos demonstrativos publicados entre os anos 1990 e 2004.

Na mesma direção, Ferrari, Kremer e Pinheiro (2013) testaram o comportamento dos custos em relação às mudanças regulatórias nas empresas do segmento de Telecomunicações da BM\&FBOVESPA, entre 1995 e 2012, confirmando que as despesas de vendas e CPV tiveram maiores variações no período de permissão de entrada de novas empresas, assim como os custos totais.

Zaro e Zaro (2013) verificaram que a adoção da certificação ISO 14001 impactou nos custos das empresas do setor petroquímico listadas na BM\&FBOVESPA, entre os anos 1994 e 2012, fazendo com que os custos aumentassem mais que a RLV, reduzindo, assim, a eficiência produtiva das empresas. 
Silva, Leal e Trindade (2015) estudaram o comportamento dos custos no setor de Carnes e Derivados e constataram que cerca de $76 \%$ da RLV é consumida pelo CPV, verificando ainda forte correlação entre as duas variáveis.

Conforme exposto, vários estudos foram realizados com base na teoria dos Sticky Costs, a fim de testar a sua aplicabilidade no Brasil. Richartz e Borgert (2014) testaram as empresas listadas na BM\&FBOVESPA em determinado período e Richartz et al. (2012) analisaram o segmento de fios e tecidos. Ambos os estudos encontraram resultados semelhantes, ou seja, a assimetria dos custos é parcialmente aplicável no Brasil, uma vez que, para variações na receita de até $10 \%$ e $30 \%$, respectivamente, a teoria se confirma. Entretanto, para variações maiores de $10 \%$ e $30 \%$, a teoria é rejeitada, e o que se observa é o inverso, ou seja, comportamento Anti-Sticky. Medeiros et al. (2005) também encontraram como resultado a parcialidade de aplicação no Brasil, pois, quando considerados períodos defasados, houve reversão parcial do comportamento assimétrico.

O estudo de Lopes e Beuren (2015), realizado no setor aéreo brasileiro, e o de Richartz et al. (2014), que buscou analisar as empresas brasileiras listadas na BM\&FBOVESPA, confirmaram a teoria dos Sticky Costs. A influência na relação de assimetria com outras variáveis, no caso do primeiro estudo, foi confirmada, porém, para o segundo estudo, não se encontrou essa influência.

Também foi confirmada a teoria dos Sticky Costs nos artigos de Fazoli et al. (2015) e Costa et al. (2013), os quais analisaram o setor industrial de Santa Catarina e as empresas abertas de nove países da América Latina, respectivamente. No setor industrial, ficou comprovada a assimetria também na separação das empresas por segmentos, tendo a maioria dos segmentos se apresentado assimétrica. Portanto, com base nos estudos realizados, pode-se inferir que a teoria dos Stick Costs é aplicável às empresas brasileiras.

Internacionalmente, Banker, Byzalov e Plehn-Dujowich (2013) examinaram a incerteza da demanda com o comportamento assimétrico dos custos, considerando duas amostras, entre os anos 1958 e 2008. Os resultados indicam que, em média, 
quando as vendas aumentam em $1 \%$, o número de empregados aumenta em $0,53 \%$, a folha de pagamento em $0,59 \%$, o número de trabalhadores da produção em 0,56\%, o número de trabalhadores administrativos em $0,44 \%$, as horas de produção em $0,59 \%$, o custo dos materiais em $0,88 \%$, o custo da energia em $0,43 \%$, despesas em $0,61 \%$ e o CPV aumenta $0,93 \%$.

Banker e Byzalov (2014) testaram o modelo proposto por Anderson, Banker e Janakiraman (2003) e Banker et al. (2013) para testar hipóteses com dados anuais de empresas de diversos países e identificaram que a assimetria dos custos é um fenômeno global.

Verifica-se que poucas pesquisas trataram de segmentos específicos a fim de analisar o comportamento dos custos. Assim, espera-se que o presente estudo contribua com a literatura analisando o comportamento dos custos no segmento de energia elétrica.

\section{PROCEDIMENTOS METODOLÓGICOS}

Quanto aos objetivos, a presente pesquisa classifica-se como descritiva. Foram descritas as características do comportamento dos custos das empresas do segmento de energia elétrica listadas na BM\&FBOVESPA.

Quanto à abordagem, utilizou-se o método quantitativo, com a aplicação de métodos estatísticos, tanto na coleta quanto no tratamento dos dados (Richardson, 2014).

Quanto aos procedimentos técnicos, adotou-se o levantamento, uma vez que foram coletadas informações a fim de compreender o comportamento dos custos em relação às receitas.

Para a coleta de dados, utilizou-se a base de dados do Economática, tendo sido coletadas informações dos demonstrativos financeiros das empresas listadas na BM\&FBOVESPA do segmento de energia elétrica, no período de 2006 a 2015. 
Para a realização da pesquisa, foram escolhidas as empresas listadas na BM\&FBOVESPA, no segmento de Energia Elétrica, o qual integra o setor de Utilidade Pública que é composto pelos segmentos de Água e Saneamento e Gás. A população da pesquisa é composta por 61 empresas listadas no segmento de energia elétrica. Para a delimitação da amostra da pesquisa, foram selecionadas 22 empresas que apresentaram algum nível de governança corporativa. Segundo Vieira e Mendes (2004), os níveis de governança corporativa aumentam a confiança dos acionistas, como também são eficazes na qualidade das informações fornecidas pela empresa. Espera-se, como resultado da transparência das informações, uma menor variação nos preços e maiores valorizações das ações.

Importante ressaltar que as empresas selecionadas apresentavam as seguintes classificações nos níveis de governança: Novo Mercado, cujo nível de transparência é mais elevado; o Nível II, equiparado ao Novo Mercado; e o Nível I, com menos obrigatoriedades, embora haja exigência da transparência e do acesso às informações pelos acionistas (BM\&FBOVESPA, 2016). Assim, a amostra escolhida foi de 21 empresas classificadas nos níveis de governança corporativa definidos. Contudo, 17 empresas integraram a amostra final, pois 4 não atenderam os requisitos necessários para a análise proposta. As empresas cujos nomes de pregão são Cesp, Ceee-D e Eletropaulo não apresentaram dados consolidados no período de análise e foram excluídas da amostra final. A CPFL Renovav que, embora tenha apresentado dados em todos os anos, é controlada por uma das empresas participantes da amostra, a CPFL Energia. Assim, como os dados coletados são consolidados, a CPFL Renovav participa da pesquisa, sendo parte do grupo.

A Tabela 2 apresenta as empresas participantes da amostra da pesquisa. 
Comportamento dos Custos das Empresas Brasileiras do Segmento de Energia Elétrica Listadas na BM\&FBOVESPA entre o Período de 2006 e 2015 Monalisa de Castro Souza, Edvalda Araújo Leal

Tabela 2

Composição da amostra da pesquisa

\begin{tabular}{|c|c|c|c|c|}
\hline Ordem & $\begin{array}{l}\text { Nome de } \\
\text { Pregão }\end{array}$ & Razão Social & Segmento & UF \\
\hline 1 & AES TIETE E & AES TIETE ENERGIA SA & N2 & SP \\
\hline 2 & ALUPAR & ALUPAR INVESTIMENTO S/A & N2 & SP \\
\hline 3 & CEEE-GT & $\begin{array}{l}\text { CIA. ESTADUAL GER.TRANS.ENER.ELET-CEEE- } \\
\text { GT }\end{array}$ & $\mathrm{N} 1$ & RS \\
\hline 4 & CELESC & CENTRAIS ELET. DE SANTA CATARINA S.A. & $\mathrm{N} 2$ & SC \\
\hline 5 & CEMIG & CIA. ENERGETICA DE MINAS GERAIS - CEMIG & $\mathrm{N} 1$ & MG \\
\hline 6 & COPEL & CIA. PARANAENSE DE ENERGIA - COPEL & N1 & PR \\
\hline 7 & $\begin{array}{l}\text { CPFL } \\
\text { ENERGIA }\end{array}$ & CPFL ENERGIA S.A. & NM & SP \\
\hline 8 & ELETROBRAS & CENTRAIS ELET. BRAS S.A. - ELETROBRAS & N1 & DF \\
\hline 9 & $\begin{array}{l}\text { ENERGIAS } \\
\text { BR }\end{array}$ & EDP - ENERGIAS DO BRASIL S.A. & NM & SP \\
\hline 10 & ENERGISA & ENERGISA S.A. & N2 & MG \\
\hline 11 & ENEVA & ENEVA S.A. & NM & RJ \\
\hline 12 & $\begin{array}{l}\text { ENGIE } \\
\text { BRASIL }\end{array}$ & ENGIE BRASIL ENERGIA S.A. & NM & SC \\
\hline 13 & EQUATORIAL & EQUATORIAL ENERGIA S.A. & NM & MA \\
\hline 14 & LIGHT S/A & LIGHT S.A. & NM & RJ \\
\hline 15 & RENOVA & RENOVA ENERGIA S.A. & N2 & SP \\
\hline 16 & TAESA & $\begin{array}{llll}\text { TRANSMISSORA } & \text { ALIANÇA } & \text { DE } & \text { ENERGIA } \\
\text { ELÉTRICA S.A. } & & & \end{array}$ & N2 & RJ \\
\hline 17 & $\begin{array}{l}\text { TRAN } \\
\text { PAULIST }\end{array}$ & $\begin{array}{l}\text { CTEEP - CIA TRANSMISSÃO ENERGIA ELÉTRICA } \\
\text { PAULISTA }\end{array}$ & $\mathrm{N} 1$ & SP \\
\hline
\end{tabular}

Nota. Fonte: Elaborada pela autora, com base nos dados de pesquisa.

Importante destacar que algumas empresas da amostra não apresentaram dados em todo o período de análise da pesquisa, que corresponde aos anos 2006 a 2015. A Alupar, Renova e Taesa não apresentaram as demonstrações financeiras de 2006. Já a Tran Paulist não apresentou dados referentes aos anos 2006 e 2007 e, por fim, a Ceee-Gt exibiu apenas as demonstrações financeiras de 2014 e 2015.

Com a amostra definida, foram coletadas, na base de dados do Economática, dos anos 2006 a 2015, as informações das demonstrações financeiras consolidadas referentes a: Receita Líquida de Vendas (RLV); Custo do Produto Vendido (CPV); Despesas Administrativas (DA); e Despesas de Vendas (DV). Após a coleta, os dados 
foram analisados da seguinte forma: (i) variação percentual das receitas e custos das empresas; e (ii) médias de custos de cada empresa ao longo do tempo. Cabe destacar que os valores foram corrigidos pelo índice do IGP-M da data de dezembro de 2015.

O pacote especializado SPSS foi utilizado para a realização do teste estatístico de correlação. Para analisar o comportamento dos custos e a associação com a receita líquida de venda, foi empregado o coeficiente de correlação de Spearman. Nesse sentido, realizou-se o teste de normalidade de Kolmogorov-Smirnov nos dados, que apontou não normalidade dos dados.

Utilizou-se ainda a análise de correlação com o propósito de identificar o quão forte é a associação entre as variáveis analisadas (receita de venda, custos e despesas), ou seja, o quanto uma variável pode impactar na outra (Malhotra, 2001).

A seguir, serão apresentados os resultados da pesquisa, a qual se destina a analisar o comportamento dos custos no segmento de Energia Elétrica das empresas listadas na BM\&FBOVESPA.

\section{ANÁLISE DOS RESULTADOS}

\subsection{Análise da Variação Percentual das Receitas e Custos}

Por meio dos dados coletados para a pesquisa, foi possível demonstrar as variações das receitas e dos custos ao longo do período de 2006 a 2015. Inicialmente, realizou-se a análise das variações percentuais das receitas líquidas de vendas, no período de dez anos, conforme Tabela 3. 
Comportamento dos Custos das Empresas Brasileiras do Segmento de Energia Elétrica Listadas na BM\&FBOVESPA entre o Período de 2006 e 2015 Monalisa de Castro Souza, Edvalda Araújo Leal

Tabela 3

Variações da RLV no período de 2006 a 2015

\begin{tabular}{|l|c|c|c|c|c|c|c|c|c|}
\hline \multicolumn{1}{|c|}{ Empresas } & $\mathbf{2 0 0 7}$ & $\mathbf{2 0 0 8}$ & $\mathbf{2 0 0 9}$ & $\mathbf{2 0 1 0}$ & $\mathbf{2 0 1 1}$ & $\mathbf{2 0 1 2}$ & $\mathbf{2 0 1 3}$ & $\mathbf{2 0 1 4}$ & $\mathbf{2 0 1 5}$ \\
\hline AES TIETE & $116 \%$ & $-11 \%$ & $-8 \%$ & $21 \%$ & $-11 \%$ & $-3 \%$ & $-13 \%$ & $18 \%$ & $-78 \%$ \\
\hline ALUPAR & - & $201 \%$ & $0 \%$ & $107 \%$ & $-18 \%$ & $-1 \%$ & $-5 \%$ & $8 \%$ & $-2 \%$ \\
\hline CEEE-GT & - & - & - & - & - & - & - & - & $-5 \%$ \\
\hline CELESC & $-7 \%$ & $3 \%$ & $-5 \%$ & $12 \%$ & $-7 \%$ & $3 \%$ & $-1 \%$ & $21 \%$ & $6 \%$ \\
\hline CEMIG & $2 \%$ & $-1 \%$ & $-2 \%$ & $12 \%$ & $10 \%$ & $11 \%$ & $-27 \%$ & $27 \%$ & $5 \%$ \\
\hline COPEL & $-3 \%$ & $-7 \%$ & $-6 \%$ & $25 \%$ & $1 \%$ & $4 \%$ & $0 \%$ & $44 \%$ & $2 \%$ \\
\hline CPFL ENERGIA & $2 \%$ & $-4 \%$ & $-1 \%$ & $16 \%$ & $-5 \%$ & $12 \%$ & $-10 \%$ & $12 \%$ & $13 \%$ \\
\hline ELETROBRAS & $10 \%$ & $24 \%$ & $-21 \%$ & $16 \%$ & $0 \%$ & $14 \%$ & $-34 \%$ & $2 \%$ & $4 \%$ \\
\hline ENERGIAS BR & $-5 \%$ & $1 \%$ & $-14 \%$ & $10 \%$ & $-4 \%$ & $16 \%$ & $0 \%$ & $19 \%$ & $10 \%$ \\
\hline ENERGISA & $38 \%$ & $-5 \%$ & $-3 \%$ & $25 \%$ & $1 \%$ & $14 \%$ & $-9 \%$ & $175 \%$ & $39 \%$ \\
\hline ENEVA & - & - & $618 \%$ & $57 \%$ & $54 \%$ & $178 \%$ & $172 \%$ & $18 \%$ & $-19 \%$ \\
\hline ENGIE BRASIL & $8 \%$ & $5 \%$ & $-7 \%$ & $19 \%$ & $-5 \%$ & $8 \%$ & $5 \%$ & $10 \%$ & $-3 \%$ \\
\hline EQUATORIAL & $-1 \%$ & $148 \%$ & $-3 \%$ & $-27 \%$ & $-1 \%$ & $44 \%$ & $46 \%$ & $36 \%$ & $2 \%$ \\
\hline LIGHT S/S & $-11 \%$ & $0 \%$ & $-8 \%$ & $22 \%$ & $-4 \%$ & $4 \%$ & $-10 \%$ & $18 \%$ & $11 \%$ \\
\hline RENOVA & - & - & $100 \%$ & $11 \%$ & $-11 \%$ & $201 \%$ & $81 \%$ & $27 \%$ & $31 \%$ \\
\hline TAESA & - & $12 \%$ & $12 \%$ & $8 \%$ & $12 \%$ & $17 \%$ & $10 \%$ & $-2 \%$ & $-1 \%$ \\
\hline TRAN PAULIST & - & - & $-4 \%$ & $39 \%$ & $15 \%$ & $-8 \%$ & $-68 \%$ & $7 \%$ & $13 \%$ \\
\hline
\end{tabular}

Nota. Fonte: Elaborada pela autora, com base nos dados da pesquisa.

Pode-se inferir, conforme os dados apresentados na Tabela 3, que, nos anos 2010 a 2014, as empresas apresentaram mais variações positivas da receita líquida de venda - RLV, destacando as variações de 107\% e 175\%, nas empresas Alupar e Energisa, respectivamente. $O$ ano em que ocorreram mais variações negativas foi 2009 , quando a empresa Eletrobrás apresentou 21\% de decréscimo na RLV. A maior variação negativa foi de 78\%, na empresa AES Tiete, no ano de 2015.

Destaca-se que, nos anos 2008 a 2012, ocorreram variações de mais de 147\% da RLV e, no ano de 2013, as empresas Cemig, Eletrobrás e Tran Paulist apresentaram variações negativas de $27 \%$, $34 \%$ e $68 \%$, respectivamente.

Cabe salientar que algumas empresas não apresentaram dados em suas demonstrações financeiras; portanto, não tiveram variações. São elas: Alupar, Renova 
e Taesa, no ano de 2007, e Tran Paulist, nos anos de 2007 e 2008. A empresa Ceee-Gt apresentou variações somente no ano de 2015.

A Eneva foi a empresa que apresentou maior crescimento da RLV no período analisado, evidenciando, no ano de 2009 , uma variação de $618 \%$ de RLV. Importante destacar que essa empresa não teve receita e CPV nos anos 2006 e 2007, não apresentando variação nos anos 2007 e 2008. A Renova não obteve receita e custo no ano de 2007, porém se destacou com uma das maiores variações de RLV no período. As empresas Ceee-Gt e Trans Paulist apresentaram decréscimo de RLV no período de 10 anos.

Portanto, pode-se verificar que a empresa com maior crescimento da RLV, no período de 2006 a 2015, foi a Eneva. Outra empresa que apresentou variação média negativa da RLV ao longo do período analisado foi a Tran Paulist. Destaca-se que as duas empresas não obtiveram RLV nos anos de 2006 e 2007.

A Tabela 4 mostra as variações dos Custos no período de 2006 a 2015. 
Comportamento dos Custos das Empresas Brasileiras do Segmento de Energia Elétrica Listadas na BM\&FBOVESPA entre o Período de 2006 e 2015 Monalisa de Castro Souza, Edvalda Araújo Leal

Tabela 4

Variações do CPV no período de 2006 a 2015

\begin{tabular}{|l|c|c|c|c|c|c|c|c|c|}
\hline \multicolumn{1}{|c|}{ Empresas } & $\mathbf{2 0 0 7}$ & $\mathbf{2 0 0 8}$ & $\mathbf{2 0 0 9}$ & $\mathbf{2 0 1 0}$ & $\mathbf{2 0 1 1}$ & $\mathbf{2 0 1 2}$ & $\mathbf{2 0 1 3}$ & $\mathbf{2 0 1 4}$ & $\mathbf{2 0 1 5}$ \\
\hline AES TIETE & $123 \%$ & $55 \%$ & $-8 \%$ & $20 \%$ & $-11 \%$ & $16 \%$ & $-17 \%$ & $36 \%$ & $-88 \%$ \\
\hline ALUPAR & - & $140 \%$ & $-2 \%$ & $414 \%$ & $-46 \%$ & $-26 \%$ & $-17 \%$ & $27 \%$ & $-5 \%$ \\
\hline CEEE-GT & - & - & - & - & - & - & - & - & $-42 \%$ \\
\hline CELESC & $5 \%$ & $10 \%$ & $1 \%$ & $15 \%$ & $-13 \%$ & $20 \%$ & $-8 \%$ & $22 \%$ & $18 \%$ \\
\hline CEMIG & $-13 \%$ & $0 \%$ & $-1 \%$ & $14 \%$ & $8 \%$ & $18 \%$ & $-26 \%$ & $23 \%$ & $19 \%$ \\
\hline COPEL & $2 \%$ & $3 \%$ & $-1 \%$ & $34 \%$ & $-1 \%$ & $15 \%$ & $-1 \%$ & $50 \%$ & $2 \%$ \\
\hline $\begin{array}{l}\text { CPFL } \\
\text { ENERGIA }\end{array}$ & $-9 \%$ & $9 \%$ & $5 \%$ & $13 \%$ & $-8 \%$ & $20 \%$ & $-7 \%$ & $18 \%$ & $18 \%$ \\
\hline $\begin{array}{l}\text { ELETROBRA } \\
\text { S }\end{array}$ & - & - & - & - & $-1 \%$ & $10 \%$ & $-26 \%$ & $256 \%$ & $0 \%$ \\
\hline $\begin{array}{l}\text { ENERGIAS } \\
\text { BR }\end{array}$ & $-6 \%$ & $-1 \%$ & $-18 \%$ & $13 \%$ & $-3 \%$ & $35 \%$ & $-6 \%$ & $28 \%$ & $9 \%$ \\
\hline ENERGISA & $22 \%$ & $9 \%$ & $3 \%$ & $32 \%$ & $0 \%$ & $15 \%$ & $-8 \%$ & $203 \%$ & $56 \%$ \\
\hline ENEVA & - & - & $566 \%$ & $102 \%$ & $26 \%$ & $247 \%$ & $134 \%$ & $-1 \%$ & $-32 \%$ \\
\hline $\begin{array}{l}\text { ENGIE } \\
\text { BRASIL }\end{array}$ & $-5 \%$ & $4 \%$ & $12 \%$ & $31 \%$ & $-11 \%$ & $9 \%$ & $27 \%$ & $29 \%$ & $-8 \%$ \\
\hline EQUATORIAL & $-4 \%$ & $201 \%$ & $-1 \%$ & $-28 \%$ & $4 \%$ & $58 \%$ & $60 \%$ & $31 \%$ & $4 \%$ \\
\hline LIGHT S/S & $-17 \%$ & $-5 \%$ & $-5 \%$ & $24 \%$ & $3 \%$ & $7 \%$ & $-15 \%$ & $25 \%$ & $21 \%$ \\
\hline RENOVA & - & - & $84 \%$ & $4 \%$ & $-17 \%$ & $327 \%$ & $86 \%$ & $36 \%$ & $46 \%$ \\
\hline TAESA & - & $54 \%$ & $16 \%$ & $-70 \%$ & $-18 \%$ & $62 \%$ & $86 \%$ & $-26 \%$ & $-33 \%$ \\
\hline $\begin{array}{l}\text { TRAN } \\
\text { PAULIST }\end{array}$ & - & - & $3 \%$ & $175 \%$ & $25 \%$ & $-10 \%$ & $-58 \%$ & $-9 \%$ & $2 \%$ \\
\hline
\end{tabular}

Nota. Fonte: Elaborada pela autora, com base nos dados da pesquisa.

Com base nos dados, verifica-se que, na maior parte do período analisado, as variações dos custos foram correlatas com as variações da receita, visto que, se a RLV crescia, o CPV crescia, e o inverso também ocorria: um decréscimo na RLV ocasionava um decréscimo no CPV. Pode-se citar que as empresas Renova e Eletrobrás apresentaram convergência nas variações da RLV e do CPV. A Renova, no ano de 2012, apresentou acréscimo de $201 \%$ na RLV, enquanto o CPV variou $327 \%$. A Eletrobrás obteve queda na RLV de $34 \%$ e decréscimo de $26 \%$ no CPV no ano de 2013. Cabe destacar que, no ano de 2015 , todas as empresas apresentaram variações correlativas. 
Importante citar que, principalmente nos anos 2008 e 2009, algumas variações do CPV foram divergentes da variação da $R L V$, ou seja, uma variação negativa na $R L V$ acarretou um aumento na variação do CPV. As empresas AES Tiete e Engie Brasil registraram queda na RLV e aumento do CPV. Já a empresa AES Tiete, no ano de 2008, apresentou decréscimo na variação da RLV de 11\%, enquanto a empresa Engie Brasil apresentou variação de $-7 \%$ da RLV no ano de 2009 , sendo as variações do CPV da ordem de $55 \%$ e $12 \%$, respectivamente.

Entretanto, os resultados indicam que prevaleceu, na maioria das empresas, a convergência das variações entre RLV e CPV, tendo predominado a variação positiva, ou seja, um acréscimo na variação da RLV acarretou aumento na variação do CPV. Esse resultado converge para a teoria dos Sticky Costs, a qual enfatiza que um aumento na receita acarreta um aumento maior nos custos. Por outro lado, os custos diminuem com menor intensidade quando a receita diminui na mesma proporção. (Anderson et al., 2003).

As variações da RLV acarretaram acréscimos ou decréscimos do CPV. Assim, buscou-se analisar o comportamento dos custos, baseando-se na variação da receita, ou seja, não se buscou entender a causa nem a direção dessas variações.

\subsection{Análise das Médias de Custos de Empresas do Segmento de Energia Elétrica}

Neste tópico, destacam-se as médias dos custos e despesas em relação à Receita Líquida de Vendas - RLV (Tabela 5). Conforme mencionado na metodologia, o número de empresas que compõem as médias em cada ano não é igual, pois algumas empresas apresentaram dados incompletos ao longo do período analisado. Assim, somente os anos 2014 e 2015 apresentam-se completos, ou seja, com todas as empresas da amostra. 
Comportamento dos Custos das Empresas Brasileiras do Segmento de Energia Elétrica Listadas na BM\&FBOVESPA entre o Período de 2006 e 2015 Monalisa de Castro Souza, Edvalda Araújo Leal

Tabela 5

Médias dos custos e despesas em relação à $R L V$

\begin{tabular}{|c|c|c|c|c|}
\hline Ano & № empresas & Média CPV/RLV & Média DV/RLV & Média DA/ RLV \\
\hline $\mathbf{2 0 0 6}$ & 12 & 0,495 & 0,068 & 0,099 \\
\hline $\mathbf{2 0 0 7}$ & 15 & 0,411 & 0,052 & 0,100 \\
\hline $\mathbf{2 0 0 8}$ & 16 & 0,525 & 0,020 & 0,584 \\
\hline $\mathbf{2 0 0 9}$ & 16 & 0,535 & 0,019 & 0,276 \\
\hline $\mathbf{2 0 1 0}$ & 16 & 0,586 & 0,016 & 0,253 \\
\hline $\mathbf{2 0 1 1}$ & 16 & 0,558 & 0,016 & 0,258 \\
\hline $\mathbf{2 0 1 2}$ & 16 & 0,616 & 0,015 & 0,177 \\
\hline $\mathbf{2 0 1 3}$ & 16 & 0,616 & 0,014 & 0,137 \\
\hline $\mathbf{2 0 1 4}$ & 17 & 0,687 & 0,009 & 0,097 \\
\hline $\mathbf{2 0 1 5}$ & 17 & 0,644 & 0,009 & 0,121 \\
\hline GERAL & & $\mathbf{0 , 5 6 7}$ & $\mathbf{0 , 0 2 4}$ & $\mathbf{0 , 2 1 0}$ \\
\hline
\end{tabular}

Nota. Fonte: Elaborada pela autora, com base nos dados da pesquisa.

Com base na Tabela 5, pode-se inferir que, em média, 56\% da Receita Líquida de Vendas - RLV são destinados a cobrir o CPV no período de 2006 a 2015, para as empresas analisadas no segmento de energia elétrica. Esses resultados são incompatíveis com os resultados encontrados por Richartz et al. (2012), que analisaram as empresas do segmento de Fios e Tecidos e constataram que o CPV consome, em média, $78 \%$ da RLV.

A mesma incompatibilidade ocorre com o segmento de carnes e derivados, visto que Silva, Leal e Trindade (2015) encontraram que, em média, 76\% da RLV são absorvidos pelo CPV e também, com os resultados de Richartz e Borgert (2014), que analisaram as empresas listadas na BM\&FBOVESPA, em diferentes setores, e constataram que, em média, $71,25 \%$ do CPV consome a receita líquida de vendas.

Analisando as despesas, destaca-se que, em média, 2\% das despesas com vendas (DV) e $21 \%$ das despesas administrativas (DA) são consumidas pela RLV. O estudo de Richartz et al. (2012) se assemelha ao resultado encontrado nesta pesquisa, 
se forem somadas as despesas em que as DV representaram, em média, 10\%, e as DA, em média, $15 \%$ da RLV.

\subsection{Análise da Correlação}

Para a análise da associação entre duas variáveis e sua influência em tal relação, utilizou-se a correlação de Spearman. A Tabela 7 apresenta os resultados da correlação das variáveis: Receita Líquida de Venda, Custo do Produto Vendido, Despesa de Venda e Despesa Administrativa.

Tabela 7

\section{Correlação de Spearman}

\begin{tabular}{|c|c|c|c|c|}
\hline Variáveis & RLV & CPV & DV & DA \\
\hline RLV & 1 & & & \\
\hline CPV &, $851^{*}$ & 1 & & \\
\hline DV &, $505^{*}$ &, $628^{*}$ & 1 & \\
\hline DA &, $760^{*}$ &, $630^{*}$ &, $520^{*}$ & 1 \\
\hline
\end{tabular}

*Significativo ao nível de 0,01\%

RLV: Receita Líquida de Vendas

CPV: Custo do Produto Vendido

DV: Despesa de Venda

DA: Despesa Administrativa

Nota. Fonte: Elaborada pela autora com base nos dados da pesquisa.

Os resultados evidenciam que a RLV apresenta correlação positiva com as demais variáveis, quais sejam, CPV, DV e DA. Entre as variáveis RLV e CPV, a correlação está acima de $80 \%$, indicando forte correlação, ou seja, um aumento na RLV tende a ser seguido pelo CPV na mesma proporcionalidade. Já as variáveis DV e DA apresentaram correlação moderada com a RLV, indicando que um aumento da RLV não é seguido na mesma proporção pelas despesas. Moderadamente, também é representada a correlação entre o CPV e as despesas. Importante ressaltar que todas as correlações foram significativas ao nível de 1\% (Tabela 7). Assim, os resultados evidenciam a associação da receita e os gastos analisados. 
No estudo realizado por Silva, Leal e Trindade (2015), com as empresas do segmento de Carnes e Derivados, os resultados indicaram forte correlação entre as variáveis RLV e CPV, DV e DA. O CPV, em relação às despesas de vendas e administrativas, também se apresentou forte.

\section{CONSIDERAÇÕES FINAIS}

O aumento da competitividade no Brasil, devido à entrada de concorrentes estrangeiros, tem estimulado o acompanhamento dos custos operacionais por parte das empresas, visto que os custos são uma fonte de informação imprescindível à tomada de decisão (Lima, Egito, \& Silva, 2004). Logo, o grande desafio das empresas, na atualidade, é oferecer qualidade aos clientes e controlar seus custos operacionais, a fim de obterem lucro e perpetuarem seus empreendimentos.

Nesse cenário, o objetivo geral desta pesquisa foi verificar o comportamento dos custos em relação às receitas líquidas de vendas das empresas brasileiras do segmento de energia elétrica listadas na BM\&FBOVESPA, no período de 2006 a 2015.

A análise da variação percentual indica crescimento da receita líquida de venda das empresas no período analisado, destacando-se os anos 2010 e 2014, que apresentaram mais variações positivas em relação aos demais anos. Destaque também cabe para a empresa Eneva, cujo crescimento da RLV no período foi o maior, apresentando, em 2009, um acréscimo na RLV de 618\%. As empresas que apresentaram, na média dos 10 anos analisados, decréscimos nas variações da RLV foram a Ceee-Gt e a Tran Paulist.

Verificou-se ainda que as variações da RLV e do CPV foram correlatas na maioria das empresas investigadas, bem como positivas, ou seja, aumentos da RLV ocasionaram aumentos do CPV. Os resultados tendem para a teoria dos Stikcy Costs, em que os custos aumentam mais quando as receitas aumentam, mas diminuem quando a RLV diminui na mesma proporção (Anderson et al., 2003). 
No segmento de energia elétrica, em média, $56 \%$ da RLV são absorvidos pelo CPV. É relevante mencionar que esse resultado não foi convergente com os resultados evidenciados em outros setores analisados, visto que empresas do setor de Carnes e Derivados, empresas do segmento de Fios e Tecidos e empresas de diversos setores, listadas na BM \& FBOVESPA (Richartz et al., 2012; Richartz \& Borgert, 2014; Silva, Leal, \& Trindade, 2015) apresentaram uma parcela maior do CPV em relação à RLV, uma média de $75 \%$.

Identificou-se uma correlação forte e positiva entre a variável receita líquida de vendas - RLV e custo do produto vendido - CPV, o que indicou que, no segmento energia, os aumentos da RLV têm associação com aumentos no CPV.

A contribuição do presente estudo reforça os achados de outras pesquisas que analisaram o comportamento dos custos em outros segmentos. Verificou-se a relação significativa entre as receitas e custos das empresas do setor de energia, indicando que tais variações deverão ser analisadas no processo decisório de tais organizações.

Importante indicar que uma limitação deste estudo refere-se à análise dos dados disponibilizados nos relatórios financeiros, ou seja, não foram analisadas as informações detalhadas na composição dos custos. Para tanto, é necessária uma abertura de dados das empresas.

Torna-se relevante indicar que o presente estudo analisou o segmento de energia elétrica, portanto os resultados encontrados e expostos não podem ser generalizados. Para futuras pesquisas, sugere-se verificar se a teoria dos Sticky Costs é aplicável ao segmento de energia elétrica, bem como analisar o comportamento dos custos em outros segmentos para a comparação dos resultados obtidos neste trabalho.

\section{REFERÊNCIAS}

Agência Nacional de Energia Elétrica. (2015a). Site Institucional. Recuperado de $<$ http://www.aneel.gov.br/a-aneel>.

Agência Nacional de Energia Elétrica. (2015b). Boletim de Informações Gerenciais. Recuperado 
<http://www.aneel.gov.br/documents/656877/14854008/Boletim+de+Informa\%C3\% A7\%C3\%B5es+Gerenciais+-+Dezembro+2015/74ec6b73-0a7d-459b-b9cabb47ddb14e88>.

Agência Nacional de Energia Elétrica (2015c). Banco de Informações de Geração. Recuperado <http://www.aneel.gov.br/aplicacoes/capacidadebrasil/capacidadebrasil.cfm>.

Anderson, M. C., Banker, R. D., \& Janakiraman, S. (2003). Are selling, general, and administrative costs "sticky"? Journal of Management Information Systems. 41(1), 47-63, mar. 2003.2 Recuperado $<$ http://citeseerx.ist.psu.edu/viewdoc/summary?doi=10.1.1.17.5331>.

Andrade, M. E. M. C. (2015, novembro). Demonstração do Valor Adicionado em empresas geradoras e transmissoras de energia elétrica. Anais do Congresso Brasileiro de Custos, Foz do Iguaçu, PR, Brasil, 22. Recuperado de $<$ http://anaiscbc.emnuvens.com.br/anais/article/view/3888>.

Banker, R. D., Byzalov, D., Ciftci, M., \& Mashruwala, R. (2012). The moderating effect of prior sales changes on asymmetric cost behavior. Social Science Research Network. . Recuperado de: <http://ssrn.com/abstract=902546>.

Banker, R. D., Byzalov, D., \& Plehn-Dujowich, J. M. (2013). Demand uncertainty and cost behavior. The Accounting Review, 89 (3), p. 839-865, maio.

Banker, R. D., \& Byzalov, D. (2014). Asymmetric cost behavior. Journal of Management Accounting Research, 26( 2), p. 43-79.

BM\&FBOVESPA. (2016). Segmentos de Listagem. Recuperado de: $<$ http://www.bmfbovespa.com.br/pt_br/listagem/acoes/segmentos-delistagem/sobre-segmentos-de-listagem>.

Brasil. (2007). Ministério De Minas E Energia - MME. Matriz Energética Nacional 2030. Recuperado de: <http://www.mme.gov.br/documents/10584/1432020/Matriz+Energ\%C3\%A9tica+Br asileira+2030+-+\%28PDF\%29/708f3bd7-f3ed-4206-a855-

$44 \mathrm{f} 6 \mathrm{~d} 4 \mathrm{db} 29 \mathrm{f} 6$ ? version $=1>$.

Costa, P. de S., Marques, A. V. C., Santos, C. K. S., \& Lima, F. D. C. (2013, novembro). Análise do comportamento assimétrico dos custos nas companhias abertas dos países da América Latina. Anais do Congresso Brasileiro de Custos, Uberlândia, MG, Brasil, $20 . \quad$ Recuperado de: $<$ http://anaiscbc.emnuvens.com.br/anais/article/view/104>. 
Duarte, S. L., Fehr, L. C. F. de A., Tavares, M., \& Reis, E. A. de (2013). Comportamento das variáveis dos custos de produção da cultura do café no período de formação da lavoura. Revista Contabilidade Vista \& Revista, 24(4), 15-33. Recuperado de: $<$ http://revistas.face.ufmg.br/index.php/contabilidadevistaerevista/article/view/1308>.

Dubois, A., Kulpa, L., \& Souza, L. E. de (2009). Gestão de custos e formação de preços. (3a ed). São Paulo: Atlas.

Fazoli, J. C., Reis, L. S., \& Borgert, A. (2015, novembro). O comportamento dos custos das indústrias do estado de Santa Catarina com ênfase na teoria dos Stickycosts. Anais do Congresso Brasileiro de Custos, Foz do Iguaçu, PR, Brasil, 22. Recuperado de: <http://anaiscbc.emnuvens.com.br/anais/article/view/4057>.

Ferrari, M. J., Kremer, A. W., \& Pinheiro, N. S. (2013, novembro). Análise do comportamento dos custos no setor de telecomunicações. Anais do Congresso Brasileiro de Custos, Uberlândia, MG, Brasil, 20. Recuperado de: <http://anaiscbc.emnuvens.com.br/anais/article/view/105>.

Gomes, I. S., Lima, D. H. S. de., \& Steppan, A. I. B. (2007, dezembro). Análise do comportamento dos custos hospitalares indiretos: uma investigação empírica do custo hospitalar de energia elétrica no setor de radioterapia da liga Norte-RioGrandense contra o câncer. Anais do Congresso Brasileiro de Custos, João Pessoa, PB, Brasil, $14 . \quad$ Recuperado de: $<$ http://anaiscbc.emnuvens.com.br/anais/article/view/1659>.

Lima, G. A. S. F. de., Egito, M. O. T. do., \& Silva, J. D. G. da (2004). Utilização de informações de custos no processo gerencial: um estudo comparativo entre a hotelaria do estado do Rio Grande do Norte e a região nordeste, sob a ótica da gestão econômico-financeira. Revista Contabilidade \& Finanças, 15 (edição Especial), 106-116. Recuperado de: $<$ http://www.scielo.br/scielo.php?script=sci_arttext\&pid=S1519$<70772004000400008>$.

Lima, D. H. S. de., Santiago, J. S., Araujo, A. O., \& Leite, P. A. M., Filho (2005, novembro). Análise do comportamento dos custos indiretos em entidades hospitalares através do modelo clássico de regressão linear normal: o caso da liga Norte-Riograndense contra o câncer. Anais do Congresso Brasileiro de Custos, Florianópolis, SC, Brasil, 9. $\quad$ Recuperado de: $<$ https://anaiscbc.emnuvens.com.br/anais/article/view/2215>.

Lopes, I. F., \& Beuren, I. M. (2015, novembro). Comportamento dos custos e sua relação com medidas não financeiras de desempenho em companhias aéreas. 
Anais do Congresso Brasileiro de Custos, Foz do Iguaçu, PR, Brasil, 22. Recuperado de: <http://anaiscbc.emnuvens.com.br/anais/article/view/3990>.

Malhotra, N. K. (2001). Pesquisa de Marketing. Porto Alegre: Bookman.

Martins, E. (2003). Contabilidade de custos. (9a ed). São Paulo: Atlas.

Medeiros, O. R. de, Costa, P. de S., \& Silva, C. A. T. (2005). Testes empíricos sobre o comportamento assimétrico dos custos nas empresas brasileiras. Revista Contabilidade \& Finanças, 16(38), 47-56, maio/ago. 2005. Recuperado de: $<$ http://www.scielo.br/scielo.php?script=sci_arttext\&pid=S151970772005000200005>.

Noreen, E. (1991) Conditions under which activity-based cost systems provide relevant costs. Journal of Management Accounting Research, 3(1), 159-168. Recuperado de: <http://fisher.osu.edu/ young.53/Noreen\%20JMAR\%201991>.

Oliveira, P. H. D., Lustosa, P. R. B., \& Sales, I. C. H. (2007). Comportamento de custos como parâmetro de eficiência produtiva: uma análise empírica da Companhia Vale do Rio Doce antes e após a privatização. Revista Universo Contábil, Blumenau, 3(3), 54-70. Recuperado de: <http://proxy.furb.br/ojs/index.php/universocontabil/article/view/597>.

Padoveze, C. L. (2009). Contabilidade gerencial: um enfoque em sistema de informação contábil. (5a ed.), São Paulo: Atlas.

Rabelo, E. C. (2012). Comportamento dos Custos em uma empresa cerâmica do sul catarinense. 2012. 195f. (Dissertação de Mestrado). Universidade Federal de Santa Catarina - UFSC, Florianópolis, SC, Brasil. Recuperado de: $<$ https://repositorio.ufsc.br/xmlui/handle/123456789/100913>.

Richardson, R. J. (2014). Pesquisa Social: métodos e técnicas. (3a ed). São Paulo: Atlas.

Richartz, F., \& Borgert, A. (2014). O comportamento dos custos das empresas brasileiras listadas na BM\&FBOVESPA entre 1994 e 2011, com ênfase nos sticky costs. Contaduría y Administración,59(4), 39-70. Recuperado de: <http://www.cya.unam.mx/index.php/cya/article/view/85/85>.

Richartz, F., Borgert, A., \& Ensslin, S. R. (2014). Comportamento dos custos: mapeamento e análise sistêmica das publicações internacionais. Sociedade, Contabilidade e Gestão, 9 (3), 92-108. Recuperado de: <http://www.atena.org.br/revista/ojs-2.2.3-06/index.php/ufrj/article/view/2437>. 
Richartz, F., Borgert, A., \& Lunkes, R. J. (2014). Comportamento assimétrico dos custos nas empresas brasileiras listadas na BM\&FBOVESPA. Advances in Scientific and Applied Accounting, 7(3), 339-361. Recuperado de: <file:///C:/Users/User/Downloads/Richartz_Borgert_Lunkes_2014_ComportamentoAssimetrico-dos-_34014\%20(3).pdf>.

Richartz, F., Borgert, A., Vicente, E. F. R., \& Ferrari, M. J. (2012, novembro). Comportamento dos custos das empresas brasileiras listadas no segmento de fios e tecidos da BM\&FBOVESPA entre 1998 e 2010. Anais do Congresso Brasileiro de Custos, Bento Gonçalves, RS, 19. Recuperado de: $<$ http://anaiscbc.emnuvens.com.br/anais/article/viewFile/341/341>.

Silva, I. F. U., Leal, E. A., \& Trindade, J. A. de S. (2015). Comportamento dos custos nas empresas listadas na BM \& FBOVESPA do segmento de carnes e derivados nos anos de 2004 a 2013. Revista ABCustos, Associação Brasileira de Custos, 10(1), 94-112. Recuperado de: <https://abcustos.emnuvens.com.br/abcustos/article/viewFile/308/152>.

Vieira, S. P., \& Mendes, A. G. S. T. (2004). Governança Corporativa: uma análise de sua evolução e impactos no mercado de capitais brasileiro. Revista do BNDES, 11(22), 103-122. Recuperado de: $<$ http://www.bndes.gov.br/SiteBNDES/export/sites/default/bndes_pt/Galerias/Arquiv os/conhecimento/revista/rev2205.pdf>.

Zaro, E. S., \& Zaro, C. S. (2013, novembro). O reflexo da implantação da ISO 14001 no comportamento dos custos das empresas do setor petroquímico da BM\&FBOVESPA. Anais do Congresso Brasileiro de Custos, Uberlândia, MG, Brasil, 20. Recuperado de: <http://anaiscbc.emnuvens.com.br/anais/article/view/114/114>.

Data de Submissão: 19/08/2017

Data de Aceite: 06/02/2018 